\title{
Evaluación del Estado del Aislamiento en Transformadores a partir de Mediciones IFRA de Alto Voltaje
}

\author{
Tibanlombo, Víctor ${ }^{1}$ (D); Ramírez, Juan ${ }^{1 *}$ iD ; Granda, Nelson ${ }^{1}$ iD ; Quilumba, Franklin ${ }^{1}$ (D)

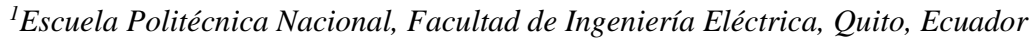

\begin{abstract}
Resumen: En este documento se presenta una evaluación experimental del estado del aislamiento de un transformador trifásico de $50 \mathrm{kVA}$ sumergido en aceite a través de la aplicación de impulsos atmosféricos estándar de alto voltaje y su respuesta en frecuencia realizando un Análisis de la Respuesta en Frecuencia al Impulso (IFRA). La evaluación se basa en tener dos estados representativos del aislamiento, por ejemplo, cuando el transformador usa aceite dieléctrico deteriorado y luego este aceite es reemplazado por uno nuevo, por lo que se realiza el cambio del aceite dieléctrico. Se lleva a cabo una valoración general del estado del transformador en ambos estados representativos mediante la ejecución de pruebas de rutina. El análisis de la respuesta en frecuencia se desarrolla mediante la obtención de la respuesta del transformador en los dos estados representativos del aislamiento y bajo diferentes configuraciones de medición. Posteriormente se evalúa a través de una comparación gráfica entre las respuestas en frecuencia, valorando cualitativamente y cuantitativamente las figuras obtenidas; y relacionándolas con el estado del aislamiento. Por ello, se proporciona una metodología experimental como una opción al uso de equipo especializado para análisis de respuesta en frecuencia por medio de la acción de impulsos atmosféricos estándar de alto voltaje, usando el equipo disponible en el Laboratorio de Alto Voltaje de la Escuela Politécnica Nacional destinado para pruebas de impulso.
\end{abstract}

Palabras clave: estado del aislamiento, transformador, impulsos atmosféricos estándar, alto voltaje, análisis de Respuesta en Frecuencia, aceite dieléctrico.

\section{Transformer Insulation Condition Assessment by Evaluation of High Voltage IFRA Measurements}

\begin{abstract}
An experimental evaluation of the insulation condition of a $50 \mathrm{kVA}$ three-phase oil-immersed transformer is presented in this document by applying high-voltage standard atmospheric impulses to obtain its frequency response, also known as Impulse Frequency Response Analysis (IFRA). The evaluation is based on comparing two different conditions of the insulation on a transformer. For instance, in this article, one insulation condition is obtained when the transformer uses deteriorated dielectric oil, and the second condition is obtained by replacing this deteriorated oil by a new one. A general evaluation of the transformer is performed in both conditions through the execution of routine tests. Frequency response analysis is implemented by obtaining the transformer response in the two insulation conditions, and under different measurement configurations. Then, the results are evaluated qualitatively and quantitatively through a graphic comparison between the measured frequency responses, and relating them to the dielectric oil insulating condition. The experimental methodology is provided as an alternative to the use of specialized frequency response analysis equipment, through the application of standard high voltage atmospheric impulses, using the equipment available in the High Voltage Laboratory of the National Polytechnic School for lightning impulse test.
\end{abstract}

Keywords: Insulation state, transformer, standard atmospheric impulses, high voltage, Frequency Response Analysis, dielectric oil.

\section{INTRODUCCIÓN}

En los últimos años el sistema eléctrico ecuatoriano ha tenido un desarrollo significativo en cuanto a expansión y transmisión, esto para satisfacer las necesidades eléctricas de una creciente población. Dentro de este desarrollo el transformador eléctrico cumple un rol importante ya que está presente en sistemas de transmisión y distribución, por ello se somete a un conjunto de pruebas tanto en fábrica para constatar calidad y ejecución de normas de diseño, como en su etapa de operación, para evaluación, registro general y constatación de funcionamiento (Valecillos B., 2005). Dentro de estas pruebas se tienen ensayos que se denominan de rutina y permiten tener una idea general del estado del transformador, específicamente 
para evaluar el aislamiento se tiene la prueba dieléctrica del aceite y la medición de resistencia del aislamiento (IEEE C57.12.90-2015, 2015). Otra prueba destinada a calificar el aislamiento ante sobrevoltajes es la prueba de impulso, la cual consiste en la inyección impulsos normalizados en los terminales de ingreso a los devanados del transformador, este ensayo es de diseño o tipo en transformadores de potencia y distribución (Valecillos B. , 2005).

Por otra parte, se tiene el Análisis de Respuesta en Frecuencia o FRA por sus siglas en inglés (Frequency Response Analysis), que es un procedimiento para evaluación del estado físico del transformador, pero también es utilizado como un apoyo a otras pruebas de diagnóstico y evaluación, permitiendo obtener una mejor idealización del estado de los elementos que conforman al transformador, entre estos se tiene el aislamiento.

Para la aplicación de un estudio FRA se utilizan equipos especializados y de un costo comercial significativo, por ejemplo: se utilizan medidores de impedancia, analizadores FRA o de barrido de frecuencia.

En este estudio se propone una opción al uso de equipo especializado para mediciones FRA, mediante el uso del equipo destinado a ensayos de impulsos atmosféricos disponible en el Laboratorio de Alto Voltaje del Departamento de Energía Eléctrica en la Escuela Politécnica Nacional (EPN) y la programación de una rutina en MATLAB para obtener resultados semejantes a un estudio FRA del transformador bajo estudio y poder relacionar el estado del aislamiento con los resultados obtenidos.

\section{MARCO TEÓRICO}

\subsection{Pruebas de rutina}

La Norma IEEE Std. C57.12.90 detalla los ensayos que se realizan a transformadores de distribución sumergidos en aceite (IEEE, 2015). A continuación, se listan las pruebas de rutina que generalmente se lleva a cabo bajo norma en el Laboratorio de Alto Voltaje de la EPN:

- Medición de la resistencia de aislamiento.

- Relación de transformación de medida.

- Prueba de circuito abierto.

- Prueba de cortocircuito.

- Prueba de voltaje aplicado.

- Prueba dieléctrica del aceite aislante.

\subsection{Análisis de respuesta en frecuencia en transformadores}

Gómez- Luna, Aponte G. y Pleite M.J (2017) en su investigación resaltan que el Análisis de Respuesta en Frecuencia es especialmente empleado para la detección de fallos mecánicos, como pueden ser desplazamientos o deformaciones en los arrollamientos o en las chapas del núcleo, los cuales son difíciles de localizar con otras metodologías.
El método FRA en transformadores se realiza inyectando por un terminal una señal de frecuencia variable o con un ancho de banda amplio obteniendo la respuesta a esta excitación en un terminal distinto, por lo cual se considera al transformador como un modelo de parámetros distribuidos, resistivos (R), inductivos (L) y capacitivos (C). Se obtiene la función de transferencia de esta red RLC mediante la relación de las señales de excitación y de respuesta (Alsuhaibani, Khan, Beroual, \& Malik, 2016).

Cualquier cambio en el interior del transformador cambiará la red RLC que lo representa (Abdallah, 2009). El método FRA se emplea para identificar estos pequeños cambios dentro del transformador al examinar la respuesta de frecuencia del transformador ya que el principal criterio de evaluación en FRA consiste en comparar las respuestas en frecuencia de diferentes estados representativos (Picher, Lapworth, Noonan, \& Christian, 2008); (Alsuhaibani, Khan, Beroual, \& Malik, 2016); (IEEE Std C57-149, 2012).

De acuerdo a la naturaleza de la señal de excitación, existen dos métodos principales de medición (Alsuhaibani, Khan, Beroual, \& Malik, 2016):

\subsubsection{Análisis de barrido en frecuencia (SFRA)}

En el método SFRA, la señal de origen o excitación es una onda sinusoidal de baja amplitud (generalmente de amplitud de 1 a 20V), que se aplica a un terminal del transformador con un barrido de frecuencia (con un rango de Hertz a Megahertz), la función de transferencia se obtiene de la relación salida / entrada para varias frecuencias (Alsuhaibani, Khan, Beroual, \& Malik, 2016).

Para la representación de mediciones SFRA, se usan todas las combinaciones de escalas gráficas lineal y logarítmica. De acuerdo con el modo de ilustración empleado se pueden obtener diferentes aspectos visuales para la representación de las curvas. La elección de la mejor visualización es esencial, ya que un estudio FRA generalmente se basa en una evaluación puramente visual de las curvas FRA (Picher, Lapworth, Noonan, \& Christian, 2008).

\subsubsection{Análisis de respuesta en frecuencia al impulso (IFRA)}

En este método se inyecta una onda no periódica como excitación o entrada en cualquier terminal, el pico máximo del impulso de entrada puede alcanzar cientos de voltios y el espectro de frecuencia se asegura mediante la forma de onda del impulso de entrada. Esto ocasiona voltajes inducidos en los extremos restantes del mismo transformador, obteniendo su respuesta (Alsuhaibani, Khan, Beroual, \& Malik, 2016).

La representación de la función de transferencia mediante el método IFRA se realiza de manera similar al SFRA, empleando gráficas lineales, logarítmicas o una combinación de éstas dependiendo de cuál visualice mejor la traza obtenida. 


\section{METODOLOGÍA}

\subsection{Transformador bajo estudio}

El transformador empleado para realizar la metodología experimental es un transformador trifásico de la marca alemana ELIN de $50 \mathrm{kVA}$ de potencia sumergido en aceite.

En la Tabla 1. se presentan las características tomadas de sus datos de placa.

Tabla 1. Datos de placa del transformador

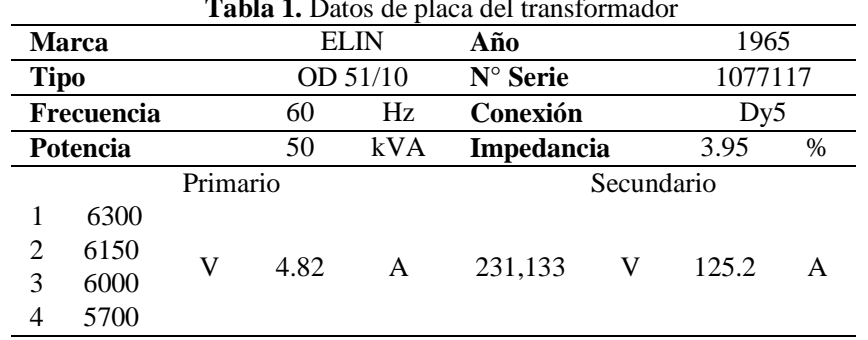

\subsection{Pruebas de rutina en el laboratorio}

Se aplicaron las pruebas de rutina mencionadas en la Sección 2 , antes y después del cambio de aceite. Las pruebas de rutina otorgan una visualización general del estado del transformador, en especial las pruebas de: resistencia de aislamiento, voltaje aplicado y prueba de aceite, que son de interés en la evaluación del aislamiento del transformador y que es parte del objetivo del presente estudio.

\subsection{Mantenimiento: Cambio del aceite dieléctrico}

Con el fin de revitalizar el aceite dieléctrico cuando sus características físicas, eléctricas y químicas se encuentran deterioradas, se realiza la regeneración o el cambio del mismo.

Las actividades sugeridas para el cambio de aceite son:

- Suministro del aceite mineral

- Desenergización del transformador

- Ensayos preliminares dieléctricos (medición de la resistencia de aislamiento y prueba dieléctrica del aceite a cambiar).

- Inspección interna (núcleo, bobinados) y ajuste de tornillería en general.

- Secado del conjunto núcleo-bobinas.

- Lavado del tanque del transformador con el nuevo aceite mineral.

- Lavado del conjunto núcleo-bobinas con el nuevo aceite mineral.

- Inyección del aceite nuevo al transformador.

- Revisión del nivel de aceite.

- Pruebas dieléctricas posteriores al cambio (medición de la resistencia de aislamiento y prueba dieléctrica del aceite).
3.4 Medición y obtención de la respuesta en frecuencia mediante impulsos estándar de alto voltaje

Para este estudio, se usa la metodología IFRA con una excitación correspondiente a un impulso atmosférico estándar con un pico máximo entre los $25 \mathrm{kV}$ y $50 \mathrm{kV}$ y una estimación aproximada del ancho de banda de la onda de $300 \mathrm{kHz}$; se puede estimar el ancho de banda mediante el tiempo de subida de la onda (Nikjoo R., 2016).

Para representar una onda de impulso atmosférico se produce una señal aperiódica que adquiere un valor pico en $1.2 \mu$ s y disminuye lentamente a la mitad de su valor pico alrededor de los $50 \mu \mathrm{s}$ (IEC, 1989). Para la generación de este tipo de impulsos el Laboratorio de Alto Voltaje de la EPN tiene un "Kit de Alto Voltaje" con el cual se puede construir un generador de impulsos atmosféricos.

La inyección del impulso se realiza de manera similar que un estudio FRA y por medio de conexiones que generalmente se aplican a transformadores sumergidos en aceite como se mencionan en la norma IEEE Std C57.149-2012.

Por otra parte, para la medición del impulso de alto voltaje se realiza mediante la combinación de un divisor capacitivo, ya que el osciloscopio necesita una señal reducida en amplitud para la seguridad de este, pues se maneja valores entre 25 y 50 $\mathrm{kV}$ de magnitud de onda.

\subsubsection{Medición de las señales de entrada y salida}

Medición de circuito abierto: La medición consiste en la inyección de la señal excitante por un terminal y la obtención de su respuesta en otro terminal diferente del mismo nivel de voltaje, con el resto de los terminales desconectados, como en el esquema de la Figura 1 (IEEE Std C57.149, 2012)

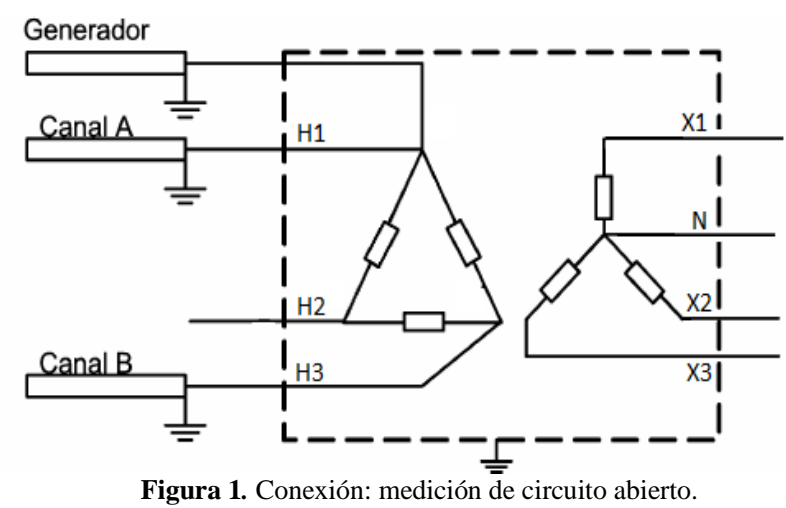

Medición de entrelazado capacitivo: La medición se realiza entre devanados de diferentes niveles de voltaje, realizando una medición capacitiva desde un extremo de un terminal a otro terminal, con todos los demás terminales desconectados, en la Figura 2, se observa un esquema de la medición. 


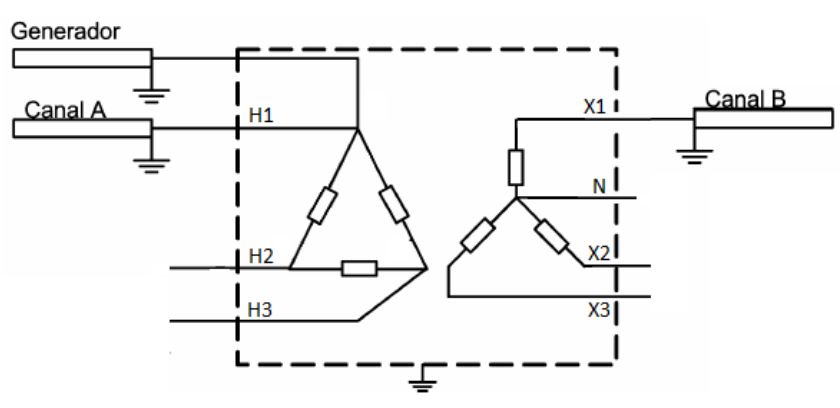

Figura 2. Conexión: medición de entrelazado capacitivo.

Medición de cortocircuito: Se realiza la medición desde el final de un devanado de alto voltaje a otro, mientras que el devanado de bajo voltaje se mantiene cortocircuitado.

Medición de entrelazado inductivo: Es similar a la prueba de entrelazado capacitivo, sin embargo, el extremo subsiguiente de cada devanado es conectado a tierra.

\subsubsection{Obtención de la respuesta en frecuencia.}

La obtención de la respuesta en frecuencia se realiza a través de la Transformada de Fourier. Se sabe que al tener un sistema con respuesta al impulso $\mathrm{h}(\mathrm{t})$, entrada $\mathrm{x}(\mathrm{t})$ y salida $\mathrm{y}(\mathrm{t})$, su entrada y salida se relacionan por la integral de convolución (Valecillos B. , 2005). En la Figura 3, se ilustra el principio de cálculo para la obtención de la función de transferencia.

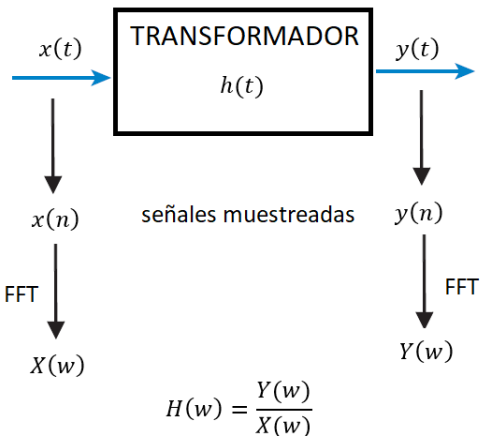

Figura 3. Principio de cálculo de la función de transferencia $H(w)$.

Valecillos. B (2005), argumenta que en un sistema la convolución en el dominio de la frecuencia representa a la multiplicación, la relación entre la entrada y salida del sistema viene dada por (1):

$$
Y(\omega)=H(\omega) X(\omega)
$$

Mediante (1) se obtiene la función de transferencia $H(\omega)$ del sistema.

Debido que al muestrear las ondas se obtiene vectores discretos, se utiliza la rutina de la Transformada Rápida de Fourier FFT presente en el entorno computacional de MATLAB, con ello se obtiene la transformada de Fourier de las señales muestreadas, el algoritmo viene dado por (2) (MathWorks, 2018).

$$
Y(k)=\sum_{n=1}^{N} x(n) e^{(-2 \pi i)(k-1)(n-1) / N}, 1 \leq k \leq N
$$

\section{$N$ : Es el número de muestras \\ $n$ : Es la n-ésima muestra original \\ $k$ : Es el k-ésimo término de la transformada}

Debido a que no se tiene un sistema ideal, se presenta ruido impuesto en las cantidades muestreadas. Para establecer un límite de frecuencia que se puede considerar "leal" a la señal obtenida antes de ser afectada por el ruido presente, se ocupa la función coherencia (Valecillos B. , 2005).

La función coherencia se obtiene con los registros en el dominio del tiempo utilizados en el cálculo de la función de transferencia. Suponiendo un comportamiento lineal del sistema examinado, y un sistema de medición ideal sin ruido, el valor debe ser igual a la unidad en toda la banda de frecuencia analizada. Ésta se promedia con diferentes impulsos inyectados con (3) (Valecillos B. , 2005).

$$
C f=\frac{\left|G_{x y}\right|^{2}}{G_{x x} G_{y y}}
$$

Donde:

$\mathrm{G}_{\mathrm{xy}}$ : Densidad cruzada de potencia espectral de las dos señales a comparar.

$\mathrm{G}_{\mathrm{xx}}$ y $\mathrm{G}_{\mathrm{yy}}$ : Densidad espectral de cada señal.

Estas densidades resultan de un promedio según el número de mediciones realizadas $y$ se obtienen mediante las transformadas en dominio de la frecuencia de las señales medidas.

\subsection{Evaluación del aislamiento a partir de pruebas IFRA}

Dentro del transformador se encuentran varias capacitancias, a saber, se tiene capacitancia entre vueltas, capacitancia entre discos, capacitancia entre devanados, y capacitancia a tierra. La capacitancia varía de acuerdo con las condiciones del conjunto papel-aceite usado como aislamiento. Esto se debe a que la permitividad relativa compleja $\varepsilon_{r}$ del papel-aceite depende del contenido de humedad y la temperatura (Yousof, Ekanayake, \& Saha, 2015). $\varepsilon_{r}$ tiene una componente compleja y real en el dominio de la frecuencia como en (4):

$$
\varepsilon_{r}=\varepsilon_{r}^{\prime}-j \varepsilon_{r}^{\prime \prime}
$$

La relación de la capacitancia compleja $\widetilde{C}$ de un material con la permitividad relativa $\varepsilon_{r}$ se obtiene con (5).

$$
\tilde{C}(\omega)=C_{0}\left\{\varepsilon_{r}^{\prime}(\omega)-j\left[\varepsilon_{r}^{\prime \prime}(\omega)+\sigma_{0} / \varepsilon_{0} \omega\right]\right\}
$$

Donde:

$C_{0}$ : Capacitancia geométrica o del vacío.

$\sigma_{0}$ : Conductividad DC del material.

$\varepsilon_{0}$ : Permitividad del vacío.

M. F. M. Yousof, C. Ekanayake and T. K. Saha (2015) proponen dos metodologías que se basan en mediciones FRA para evaluar el aislamiento:

Donde: 
1) Porcentaje de cambio de capacitancia: Desde el enfoque de FRA, la combinación paralela de una inductancia y una capacitancia crea anti-resonancia (mínimo local) o resonancia (máximo local). El cambio de capacitancia se puede calcular a partir de mediciones FRA del transformador. Para ello se puede aplicar con la ecuación de la frecuencia de resonancia de un circuito LC paralelo dado por (6).

$$
f=1 /(2 \pi \sqrt{L C})
$$

Se supone que la inductancia no presenta ningún cambio debido a que la permeabilidad magnética del conductor únicamente depende de la temperatura.

El porcentaje de cambio de capacitancia se puede calcular con (7).

$$
\Delta C_{21} \%=\left(\frac{C_{2}}{C_{1}}-1\right) \cdot 100=\left(\frac{f_{1}^{2}}{f_{2}^{2}}-1\right) \cdot 100
$$

La aplicación de (7) solo considera la parte real de la capacitancia pues la capacitancia imaginaria solo afecta la magnitud de la resonancia y no la frecuencia (Yousof, Ekanayake, \& Saha, 2015).

2) Cálculo de la capacitancia de la prueba de entrelazado capacitivo: Yousof, Ekanayake \& Saha (2015), muestran un circuito fundamental que representaría la capacitancia entre los devanados de un transformador que se mide usando la medición de entrelazado capacitivo con un analizador FRA comercial. Sin embargo, este circuito puede ser usado solo en la región de baja frecuencia. Pues la influencia de la capacitancia entre devanados estaría dominada a bajas frecuencias sin un rango definido de frecuencia. Para aplicar esta metodología en este estudio se asemeja un circuito fundamental con los equipos presentes del laboratorio para la medición.

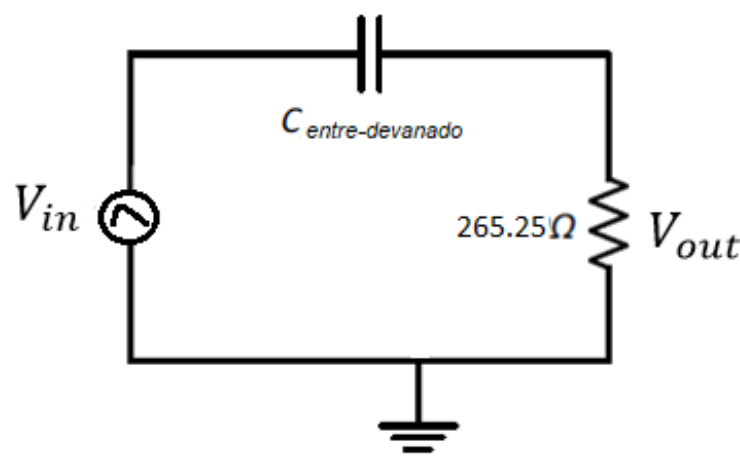

Figura 4. Conexión: medición de entrelazado capacitivo.

En la Figura 4, se representa el circuito fundamental para la representación de la capacitancia entre devanados medidas con la metodología IFRA propuesta, éste sería válido solo hasta frecuencias de alrededor de $3 \mathrm{kHz}$ según (Galván, Gutiérrez, Ortiz, \& Sánchez, 2012), de aquí se puede deducir la ecuación para el cálculo de la capacitancia entre devanados, dado por (8).

$$
\left|\frac{V_{\text {out }}}{V_{\text {in }}}\right|=\omega 265.25 \mathrm{C}
$$

\section{RESULTADOS}

\subsection{Pruebas de rutina}

Como parte del objetivo de este estudio es calificar el aislamiento del transformador bajo estudio, se aplicaron pruebas de rutina antes y después del cambio de aceite, estas son basadas en normas internacionales y realizadas con equipo presente en el laboratorio, para el fin del presente estudio se toma como relevancia los ensayos que califican el aislamiento.

\subsubsection{Medición de la resistencia de aislamiento}

Se realizó la medición basado en la norma ANSI C.57.12.90. Los resultados obtenidos se muestran en la Tabla 2.

Tabla 2. Medición de la resistencia de aislamiento

\begin{tabular}{ccccccc}
\hline Conexión & \multicolumn{2}{c}{$\begin{array}{c}\text { Voltaje } \\
\text { aplicado }\end{array}$} & \multicolumn{2}{c}{$\begin{array}{c}\text { Resistencia de aislamiento } \\
\text { Antes }\end{array}$} & \multicolumn{2}{c}{ Después } \\
\hline Alta-Baja & 5000 & $\mathrm{~V}$ & 7.14 & $\mathrm{G} \Omega$ & 12.62 & $\mathrm{G} \Omega$ \\
Alta-Tanque & 5000 & $\mathrm{~V}$ & 6.70 & $\mathrm{G} \Omega$ & 8.89 & $\mathrm{G} \Omega$ \\
Baja-Tanque & 5000 & $\mathrm{~V}$ & 82.5 & $\mathrm{M} \Omega$ & 4.25 & $\mathrm{G} \Omega$ \\
\hline
\end{tabular}

Los resultados reflejan que, al cambiar el aceite por uno nuevo, mejora notablemente ya que los valores medidos de la resistencia de aislamiento crecen, el más notable se observa en la conexión Baja-Tanque que el valor cambia de los $\mathrm{M} \Omega$ a los $\mathrm{G} \Omega$.

Se puede considerar al aceite deteriorado un buen aislante debido a que los valores están en el orden de los $M \Omega$, sin embargo, se debe tomar en cuenta que el aislamiento está formado por un conjunto de papel y aceite, por lo cual para verificar la característica del aceite se recurre a la prueba dieléctrica del aceite aislante.

\subsubsection{Prueba Dieléctrica del aceite aislante}

Los resultados se presentan en la Tabla 3, estos se comparan entre muestras obtenidas del transformador; muestras del aceite original del transformador y el nuevo aceite dieléctrico. Se realizó el ensayo bajo la norma A.S.T.M (29-D-877).

Tabla 3. Medición de la resistencia de aislamiento

\begin{tabular}{ccccc}
\hline $\mathbf{N}^{\circ}$ Prueba & Aceite original & \multicolumn{3}{c}{ Aceite nuevo } \\
\hline 1 & 12.5 & $\mathrm{kV}$ & 40.2 & $\mathrm{kV}$ \\
2 & 17.3 & $\mathrm{kV}$ & 35.5 & $\mathrm{kV}$ \\
3 & 9.1 & $\mathrm{kV}$ & 33.3 & $\mathrm{kV}$ \\
4 & 8.7 & $\mathrm{kV}$ & 32.2 & $\mathrm{kV}$ \\
5 & 10.1 & $\mathrm{kV}$ & 30.6 & $\mathrm{kV}$ \\
\hline Promedio & $\mathbf{1 1 . 5 4}$ & $\mathbf{k V}$ & $\mathbf{3 4 . 3 6}$ & $\mathbf{k V}$ \\
\hline
\end{tabular}

\subsection{Respuesta en frecuencia mediante pruebas de impulso}

Las pruebas con mejores resultados son: Prueba de circuito abierto en alto voltaje, prueba de entrelazado capacitivo y prueba de entrelazado inductivo, sin embargo, la evaluación del aislamiento se realizó a partir de las pruebas de circuito abierto y entrelazado capacitivo, los resultados se presentan a continuación: 


\subsubsection{Prueba de circuito abierto (medición en el lado de Alto Voltaje)}

Antes del cambio:

En la Figura 5, se muestra la respuesta en frecuencia de la prueba de circuito abierto, se tiene una traza similar en la forma del espectro de las tres fases de alto voltaje, por otra parte, los picos de resonancias y anti-resonancias no coinciden en las mismas frecuencias, además se tiene una diferencia en cuanto a la magnitud de éstas. Esto puede ser debido a la estructura de construcción propia del transformador.

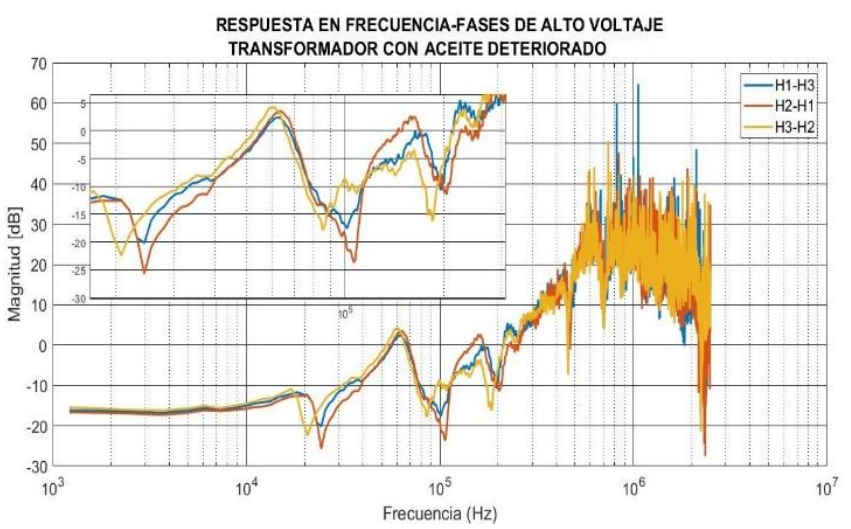

Figura 5. Respuesta en frecuencia. Tipo de prueba: OC fases de alto voltaje (H1-H3, H2-H1, H3-H2). Transformador con aceite deteriorado.

En la Figura 6, se observa la superposición entre dos respuestas de frecuencia medidas en los terminales de una misma fase, esto para el cálculo de la función coherencia a partir de dos señales de impulso.

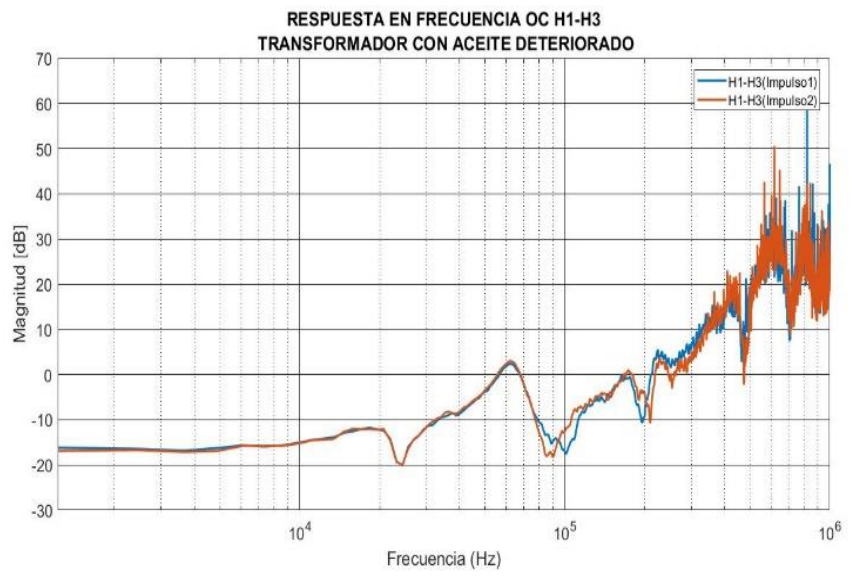

Figura 6. Respuesta en frecuencia. Tipo de prueba: OC H1-H3. Transformador con aceite deteriorado.

El cómputo de la coherencia para el sistema utilizado, se muestra en la Figura 7. Se tiene que hasta alrededor de los 180 $\mathrm{kHz}$ la respuesta es fiable, es decir que la función coherencia toma un valor cercano a 1 hasta ese valor de frecuencia, también se tiene una pequeña disminución del valor alrededor de $\operatorname{los} 100 \mathrm{kHz}$

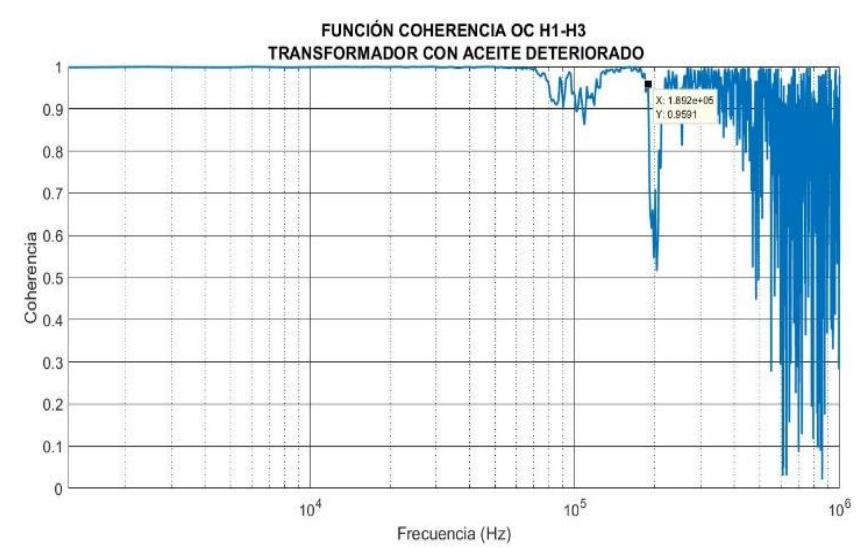

Figura 7. Coherencia. Tipo de prueba: OC H1-H3. Transformador con aceite deteriorado.

Después del cambio:

Por otra parte, la coherencia calculada para el mismo devanado H1-H3 después de cambiar el aceite dieléctrico se presenta en la Figura 8, se observa una notable mejoría, pues el valor de la función coherencia mejora hasta alrededor de los $300 \mathrm{kHz}$, también la disminución presente en los 100 kHz desaparece.

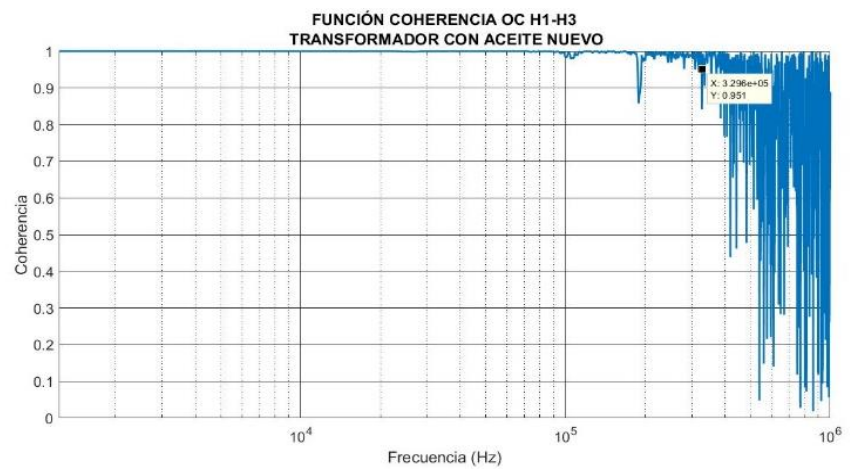

Figura 8. Coherencia. Tipo de prueba: OC H1-H3. Transformador con aceite nuevo.

Comparación de estados representativos:

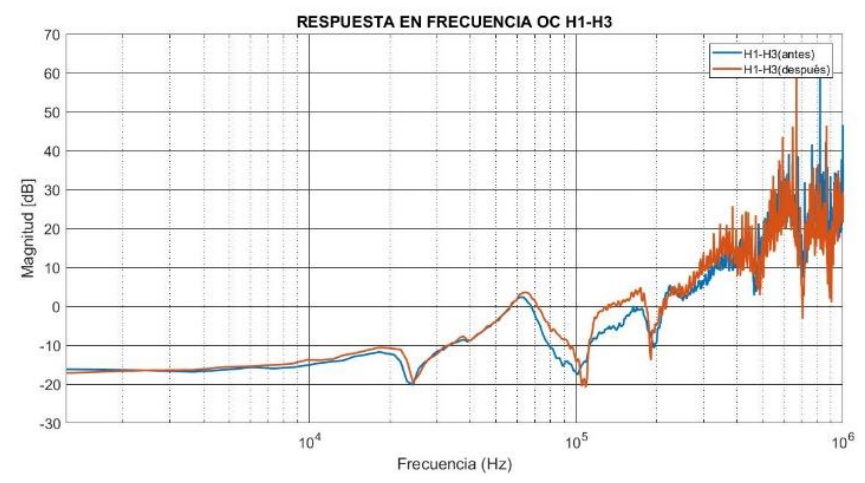

Figura 9. Comparación de estados de la respuesta en frecuencia. Transformador ELIN 50 kVA 230/6000V. Tipo de prueba: OC H1-H3.

En la Figura 9, se observa que al cambiar el aceite la respuesta en frecuencia se mueve a frecuencias mayores, pero mantiene su trazado o forma. 


\subsubsection{Prueba de Entrelazado Capacitivo}

Antes del cambio

Se observa en las gráficas obtenidas en la Figura 10 que a bajas frecuencias se tiene una linealidad con diferencia de magnitud entre las gráficas, la diferencia entre grupo de fases puede ser debido a la construcción misma de las bobinas del transformador.

Se realizó también el cálculo de la función coherencia de la respuesta obtenida bajo esta medición del transformador con dieléctrico deteriorado. El cálculo obtenido de la medición del grupo H1-X1, se observa en la Figura 11, se presenta que el valor de la función coherencia tiene un valor cercano a la unidad hasta alrededor de $40 \mathrm{kHz}$, obteniendo valores similares en los demás grupos de conexión.

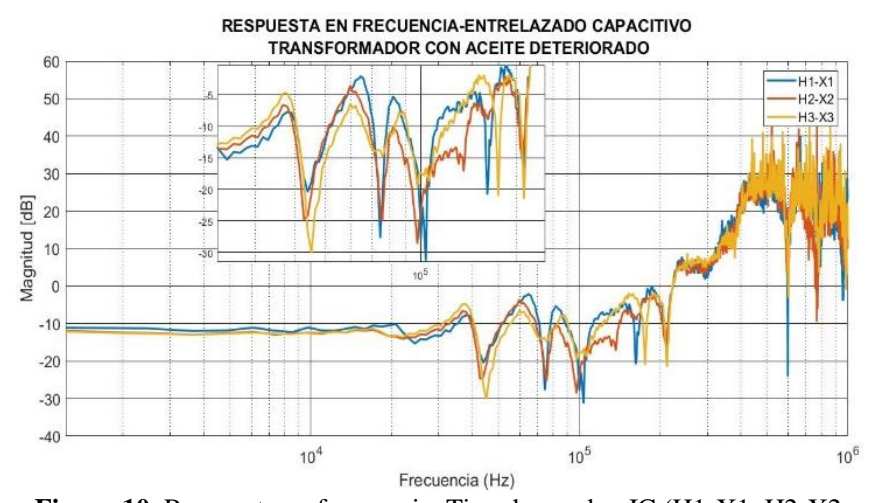

Figura 10. Respuesta en frecuencia. Tipo de prueba: IC (H1-X1, H2-X2, H3-X3). Transformador con aceite deteriorado.

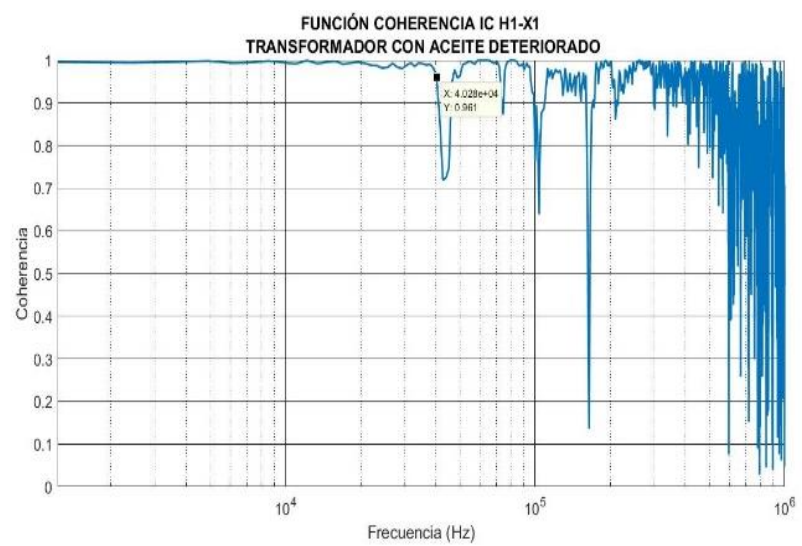

Figura 11. Coherencia. Tipo de prueba: IC H1-X1. Transformador con aceite nuevo.

Después del cambio:

La Figura 12 presenta la función coherencia del grupo H1-X1 una vez cambiado el aceite, se observa que esta tiene un valor cercano a la unidad hasta un valor de frecuencia de $300 \mathrm{kHz}$, es decir aumenta el límite de frecuencia para realizar un análisis en ese estado.

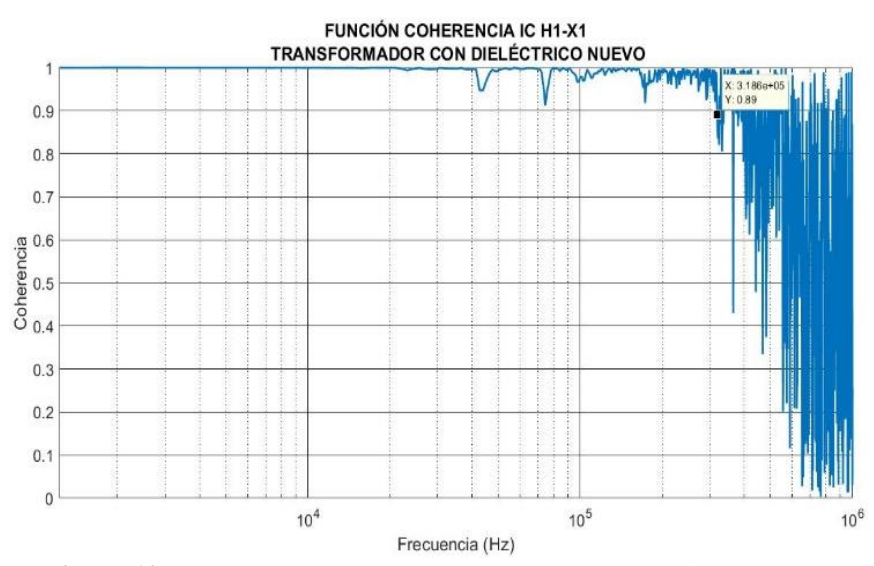

Figura 12. Coherencia. Tipo de prueba: IC H1-X1. Transformador con aceite nuevo.

Comparación de estados representativos:

En la Figura 13, se presenta las respuestas de la medición de entrelazado capacitivo para los terminales $\mathrm{H} 1-\mathrm{X} 1$, antes y después del cambio del aceite. Se presenta que la magnitud de la traza disminuye a bajas frecuencias, esto representaría un cambio en la capacitancia presente entre los devanados de alto y bajo voltaje, pues al modificar el aislamiento con el cambio de aceite, cambia la permitividad de éste.

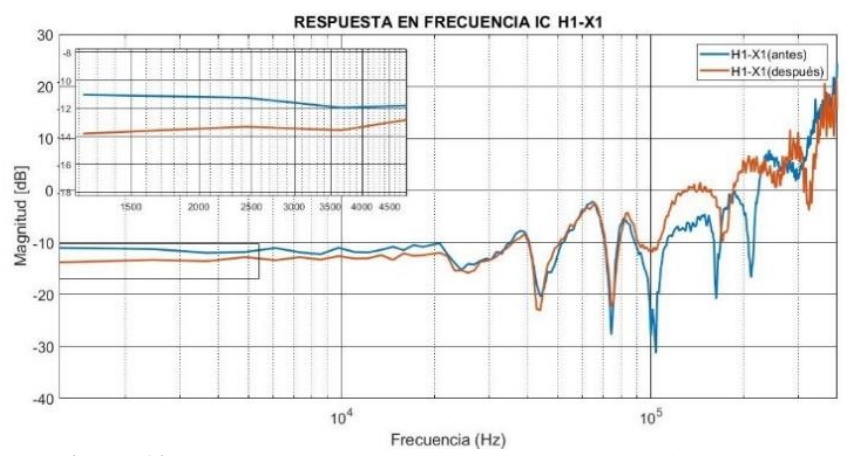

Figura 13. Comparación de estados de la respuesta en frecuencia. Transformador ELIN 50 kVA 230/6000V. Tipo de prueba: IC H1-X1.

\subsubsection{Evaluación del aislamiento a partir de las mediciones IFRA}

Para cada fase de alto voltaje se calculó el porcentaje de cambio de capacitancia $\Delta C_{21} \%$ en el aislamiento, esto partir de la respuesta en frecuencia obtenida en la medición de circuito abierto en alto voltaje, antes y después del cambio del aceite dieléctrico. Para determinar el porcentaje de cambio de capacitancia, se tomaron las frecuencias de las resonancias y las antiresonancias mostrados en la Figura 14, es decir las frecuencias con los picos máximos y mínimos. Obteniendo un promedio para el porcentaje de cambio según la Ecuación (7) en cada fase de alto voltaje, esto se observa en la Tabla 4. 


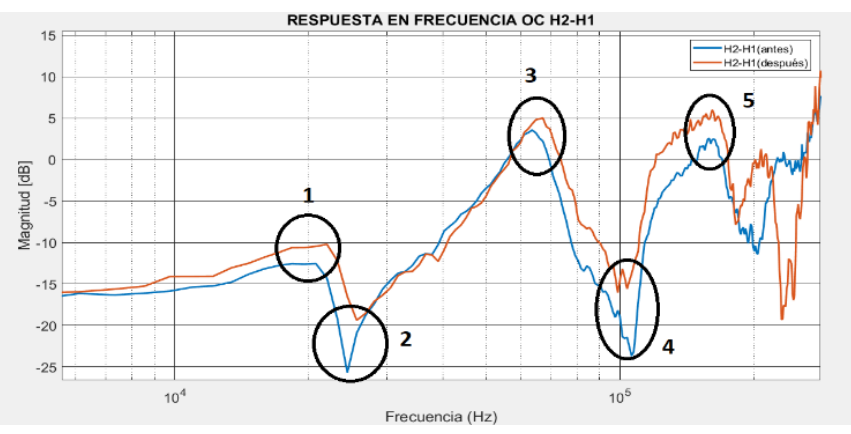

Figura 14. Respuestas en frecuencia. Transformador ELIN $50 \mathrm{kVA}$ 230/6000V. Tipo de prueba: OC H2-H1.

Se presenta que el cambio de capacitancia en cada devanado disminuye de manera diferente, esto puede ser debido a la forma de construcción de los devanados, pues las capacidades efectivas presentes entre bobinas y capas podrían variar por tener diferentes proporciones de papel o cartón, según sea el caso, también hay que tomar en cuenta que el aislamiento está conformado de un conjunto papel-aceite, por lo que el papel tendría diferentes niveles de degradación en cada bobina.

\begin{tabular}{cccc}
\multicolumn{4}{c}{ Tabla 4. Porcentaje de cambio de capacitancia } \\
\hline $\mathbf{N}^{\circ}$ & $\mathbf{H 1 - H 3}$ & $\mathbf{H 2}-\mathbf{H 1}$ & $\mathbf{H 3 - H 2}$ \\
Frecuencia & $\Delta C_{21}(\%)$ & $\Delta C_{21}(\%)$ & $\Delta C_{21}(\%)$ \\
\hline $\mathbf{1}$ & 0 & -10.797 & 0 \\
$\mathbf{2}$ & 0 & -9.29 & -10.79 \\
$\mathbf{3}$ & -7.4 & -7.136 & -3.88 \\
$\mathbf{4}$ & -12.99 & 4.677 & -17.346 \\
$\mathbf{5}$ & -12.46 & -2.95 & 3.15 \\
\hline Promedio & $\mathbf{- 6 . 5 7 \%}$ & $\mathbf{- 5 . 0 9 9 \%}$ & $\mathbf{- 5 . 7 7 3 \%}$ \\
\hline
\end{tabular}

Yousof, Ekanayake \& Saha (2015) presentan un ejemplo de esta metodología para el cálculo del porcentaje de cambio de capacitancia del aislamiento a partir de mediciones FRA de cortocircuito, ahí se evalúa el cambio producido por el envejecimiento del aislamiento, obteniendo que, al exponer el aislamiento del transformador a un proceso de degradación, el cambio en el porcentaje aumenta.

Por otra parte, se calculó un promedio de la capacitancia real mediante las mediciones de entrelazado capacitivo para cada grupo terminal y en cada estado representativo del aislamiento.

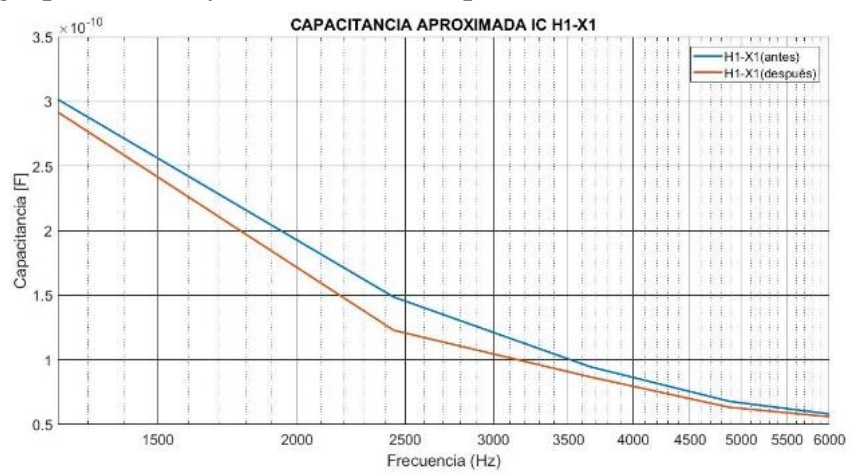

Figura 15. Capacitancia aproximada. Tipo de prueba: IC H1-X1

La Figura 15 presenta la variación obtenida de la capacitancia en función de la frecuencia sin embargo estos resultados serían válidos hasta alrededor de los $3 \mathrm{kHz}$. Los resultados del cálculo de capacitancia se presentan en la Tabla 5.
Tabla 5. Capacitancia aproximada medida

\begin{tabular}{cccc}
\hline & \multicolumn{3}{c}{ Valor promedio de capacitancia real entre } \\
& H1-X1 & H2-X2 & H3-X3 \\
\hline Antes & 1.8155 & 1.9519 & 1.9351 \\
Después & 1.6707 & 1.7373 & 1.7866 \\
\hline
\end{tabular}

En los resultados se puede apreciar que el valor de capacitancia calculado disminuye una vez que se cambia el aceite dieléctrico, es decir cuando se mejora el aislamiento, esto concuerda con el ejemplo mencionado en (Yousof, Ekanayake \& Saha, 2015), ya que, en dicho estudio, el valor de capacitancia medida aumenta al deteriorar el aislamiento, esto puede ser debido a un cambio en la permitividad del medio.

\section{CONCLUSIONES}

Se planteó una metodología práctica y experimental para la obtención de la función de transferencia del transformador bajo estudio y su respuesta en frecuencia, esto mediante la inyección de impulsos atmosféricos estándar de alto voltaje, usando los equipos e instrumentos que se tiene en el laboratorio de Alto Voltaje de la Escuela Politécnica Nacional.

La base de análisis en mediciones FRA es la comparación de curvas de respuesta en frecuencia que representen un determinado estado de la máquina bajo estudio, por ello, al comparar las figuras obtenidas en cada estado se puede encontrar diferencias notables en magnitud y frecuencia.

Al empeorar el aislamiento, se tiene un aumento en la respuesta en frecuencia IFRA calculada, esto se puede usar como un indicador de que un aislamiento está envejeciendo o deteriorándose.

El ancho de banda de un impulso atmosférico estándar está alrededor de los $300 \mathrm{kHz}$, esta banda es afectada debido al ruido presente en la digitalización de las señales y el ambiente externo, por esto se usa la función coherencia, para poder discernir un límite de frecuencia en el cual se tiene una respuesta veraz.

Se concluye que las mediciones FRA pueden ser de gran ayuda para la evaluación del aislamiento de un transformador, siendo un complemento a las pruebas de rutina que son destinadas a la evaluación de este.

\section{REFERENCIAS}

Abdallah, J. (2009). Using the Frequency Response Analysis (FRA) in Transformers Internal Fault Detection. WSEAS TRANSACTIONS on POWER SYSTEMS, 297-306.

Alsuhaibani, S., Khan, Y., Beroual, A., \& Malik, N. (2016). A Review of Frequency Response Analysis Methods for Power Transformer Diagnostics. Energies, 9,879.

Cortés, J., Medina, F., \& Chávez, J. (2007). Del Análisis de Fourier a las Wavelets. Scientia et Technica Año XIII, 13(34), 151-156. Retrieved from https://dialnet.unirioja.es/descarga/articulo/4807129.pdf.

Galván, V., Gutiérrez, J., Ortiz, V., \& Sánchez, R. (2012). Modelado del transformador para eventos de alta frecuencia. Energética.

Gómez-Luna, E., Aponte, M., \& Pleite, G. (2017). Obtención de la respuesta en frecuencia en transformadores estando en servicio. Ingeniare, 25(1), 59-69. https://dx.doi.org/10.4067/S0718-33052017000100059. 
IEC. (1989). IEC Std. 60060-1"High-voltage test techniques Part 1: General definitions and test requirements". IEC.

IEEE. (2012). IEEE Std C57-149-2012 "Guide for the Application and Interpretation of Frequency Response Analysis for Oil-Immersed Transformers". IEEE Power and Energy Society.

IEEE. (2015). IEEE C57.12.90-2015 - IEEE Standard Test Code for LiquidImmersed Distribution, Power, and Regulating Transformers. IEEE.

MathWorks. (2018, 01 25). https://www.mathworks.com. Retrieved from https://www.mathworks.com/help/matlab/ref/fft.html

Nikjoo, N., Taylor, N., Clemence, R., \& Edin, H. (2013). Dielectric Response of Aged Transformer Bushings Utilizing Power System Transients. 4th IEEE PES Innovative Smart Grid Technologies Europe (ISGT Europe). Copenhagen.

Nikjoo, R. (2016). Dielectric Response and Partial Discharge Diagnostics of Insulation Systems by Utilizing High Voltage Impulses. Stockholm: KTH Doctoral Thesis.

Picher, P., Lapworth, J., Noonan, T., \& Christian, J. (2008). MechanicalCondition Assessment of Transformer Windings Using Frequency Response Analysis (FRA). Toronto: CIGRE WG A2.26 Technical Brochure 342.

Vaessen, P., \& Hanique, E. (1992). A new frequency response analysis method for power transformers. IEEE Transactions on Power Delivery, 7(1), 384-391.

Valecillos, B. (2005). Análisis de la respuesta en frecuencia como herramienta de evaluación de las pruebas de impulso en transformadores. Madrid: Universidad Carlos III de Madrid.

Valecillos, B. (2005). Sistema de Evaluación de Resultados para los Ensayos de impulso a Transformadores. Tesis. Universidad Simón Bolívar.

Yousof, M., Ekanayake, C., \& Saha, T. (2015). Examining the ageing of transformer insulation using FRA and FDS techniques. EEE Transactions on Dielectrics and Electrical Insulation, 22(2), 1258 1265.

\section{BIOGRAFÍAS}

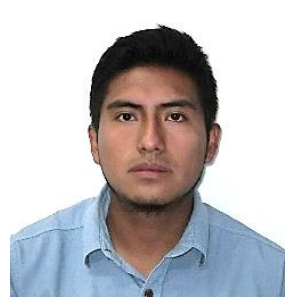

Víctor H. Tibanlombo Timbila, nació en Ambato, Ecuador el 23 de mayo de 1994. Se gradúo como Ingeniero Eléctrico en la Escuela Politécnica Nacional en el 2018. Se ha desempeñado como Ingeniero Eléctrico en proyectos de construcción de redes eléctricas de distribución urbanas y rural; proyectos en el sector petrolero del país y estudios de estabilidad.

Entre 2016 y 2018 fue asistente de cátedra en el Laboratorio de Máquinas Eléctricas en la Escuela Politécnica Nacional. Actualmente es parte del cuerpo docente en el Departamento de Energía Eléctrica de la Escuela Politécnica Nacional. Sus áreas de interés son sistemas eléctricos de potencia, ingeniería de alto voltaje y tecnologías de energías renovables.

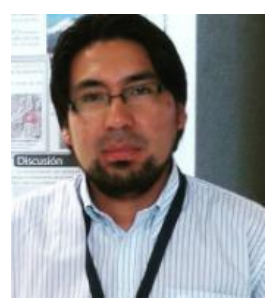

Juan D. Ramírez Guasgua, se graduó como Ingeniero Eléctrico en la Escuela Politécnica Nacional en el 2015.

Actualmente está realizando sus estudios de posgrado en Administración de Empresas de los Sectores estratégicos en la Escuela Politécnica Nacional. Se ha desempeñado como especialista técnico en la construcción de líneas de transmisión de electricidad en CELEC EP TRANSELECTRIC y en la actualidad trabaja como Profesor Ocasional en el Departamento de Energía Eléctrica de la Escuela Politécnica Nacional. Sus áreas de interés incluyen: teorías de sistemas y control aplicadas al sistema eléctrico de potencia, modelación y simulación de sistemas eléctricos, ingeniería de alto voltaje y operaciones comerciales del sector eléctrico.
Nelson V. Granda Gutiérrez, obtuvo el título de Ingeniero Eléctrico en la Escuela Politécnica Nacional en 2006 y de Doctor en Ciencias de la Ingeniería Eléctrica en la Universidad Nacional de San Juan (Argentina), en el 2015. Se ha desempeñado como Ingeniero Eléctrico en el Centro Nacional de Control de Energía (CENACE), en el departamento de Optimización de la Generación Eléctrica (OGE) de Petroamazonas EP y el la Subgerencia de Operación y Mantenimiento de CELEC-EP, TRANSELECTRIC.

Actualmente se desempeña como parte del staff docente del Departamento de Energía Eléctrica de la Politécnica Nacional. Sus áreas de interés son análisis y control de sistemas de potencia en tiempo real y aplicaciones de Sistemas de Medición de Área extendida (WAMS) basados en unidades de medición sincrofasorial (PMU).

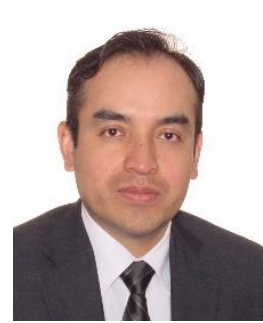

Franklin L. Quilumba Gudiño, obtuvo el título de Ingeniero Eléctrico en la Escuela Politécnica Nacional en Quito, Ecuador, en el 2008. Realizó sus estudios de posgrado en la Universidad de Texas Arlington, en Arlington, Estados Unidos de América, donde obtuvo el grado de Master of Science y el título de Doctor of Philosophy Ph.D., en Ingeniería Eléctrica, en el 2014. Entre 2014 y 2018 fue docente titular en el Departamento de Energía Eléctrica de la Escuela Politécnica Nacional. En la actualidad se desempeña como ingeniero de protecciones de sistemas de transmisión de la compañía Oncor Electric Delivery, Fort Worth, TX, USA. Sus áreas de interés incluyen modelación y análisis de sistemas eléctricos de potencia; estabilidad, protección y control de sistemas eléctricos de potencia; planificación de sistemas eléctricos de potencia. El Dr. Quilumba es Ingeniero Profesional Registrado en el Estado de Texas. 
